\title{
A Novel Multimode Waveguide Coupler for Accurate Power Measurement of Traveling Wave Tube Harmonic Frequencies
}

\author{
Edwin G. Wintucky and Rainee N. Simons \\ NASA Glenn Research Center (GRC), MS 54-1 \\ 21000 Brookpark Road, Cleveland, OH 44135, USA \\ Emails: Edwin.G.Wintucky@nasa.gov and Rainee.N.Simons@nasa.gov
}

\begin{abstract}
This paper presents the design, fabrication and test results for a novel waveguide multimode directional coupler $(M D C)$. The coupler fabricated from two dissimilar waveguides is capable of isolating the power at the second harmonic frequency from the fundamental power at the output port of a traveling-wave tube (TWT). In addition to accurate power measurements at harmonic frequencies, a potential application of the MDC is in the design of a beacon source for atmospheric propagation studies at millimeter-wave frequencies.
\end{abstract}

Keywords: TWT, waveguide coupler, harmonic frequencies

\section{INTRODUCTION}

Because of increasing congestion in available spectrum at the currently used frequency band $(3-30 \mathrm{GHz})$ for space-toground data communications, there is now an effort by NASA, other United States Government agencies and the commercial broadband satellite communications industry to develop the millimeter-wave (mm-wave) frequency band for this purpose. The mm-wave band includes frequencies in the Q-band (37-42 $\mathrm{GHz}$ ) and the V/W-band (71-76 GHz). The potential emerging applications at these frequencies include space communications for exploration missions, communications for unmanned aerial vehicles, and terrestrial broadband communications.

In this paper, we present the design, fabrication, and test results of a waveguide multimode directional coupler (MDC) [1] for the measurement of the second and higher harmonics from a high power space traveling-wave tube amplifier (TWTA) [2]. These measurements are necessary at a production facility to qualify and control the amount of interference power that a TWTA would generate. The knowledge of the amount of interference power can be factored into the interpretation and correction of the measurements from observation systems. In addition, the knowledge of the amount of interference power can be considered in improving accuracy of navigation systems. Furthermore, if the harmonic power is isolated from the fundamental it can be used as a beacon source for millimeter-wave atmospheric propagation studies needed for the design of robust space-to-ground satellite communication links [3].

\section{MDC DESIGN AND EXPERIMENTAL RESULTS}

\section{A. Waveguide Multimode Directional Coupler Design}

The waveguide MDC used in the test demonstration is designed and fabricated from two dissimilar waveguides, a larger primary waveguide for the fundamental frequency and a smaller secondary waveguide for the second harmonic as illustrated in Fig. 2. The two waveguides are joined together and share a common wall. In the primary waveguide, the signal at the fundamental frequency propagates as the dominant $\mathrm{TE}_{10}$ mode. The power in the second harmonic signal propagates as higher order modes. If an aperture is cut in the shape of a narrow rectangular slot parallel to the y-axis along the primary waveguide narrow wall the coupling to the $\mathrm{TE}_{10}$ mode will be negligibly small. However, the slot aperture will couple strongly to the $\mathrm{TM}_{11}$ type higher order mode. Thus the power in the second harmonic signal is selectively coupled to the secondary waveguide and can be amplified to a higher power level as needed.

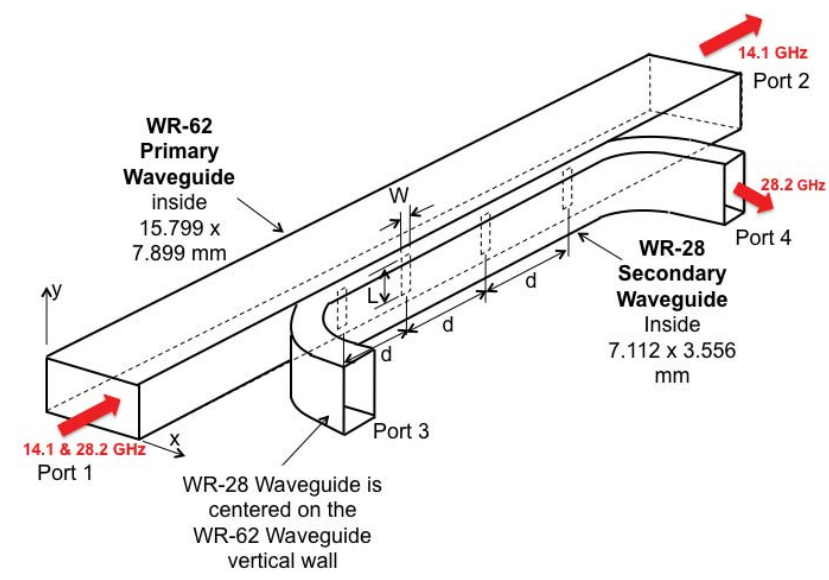

Fig. 1. Schematic of the waveguide MDC.

\section{B. Multimode Directional Coupler Fabrication}

For the initial proof-of-concept (POC) demonstration, the primary and the secondary waveguides were chosen as WR-62

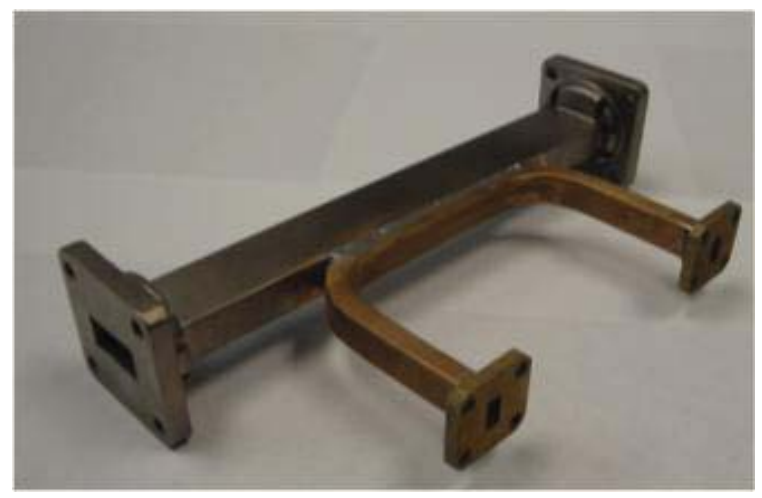

Fig. 2 Fabricated proof-of-concept Ku-band/Ka-band waveguide MDC. 
and WR-28, respectively. The fundamental frequency is at $\mathrm{Ku}-$ band (13.25-18 GHz) and the second harmonic is at Ka-band (26.5-36 GHz). Fig. 2 shows the fabricated POC MDC used in the demonstrations. Two MDCs were fabricated to enable the measurement of the coupling coefficient at the second harmonic frequencies.

\section{Multimode Directional Coupler Test Data}

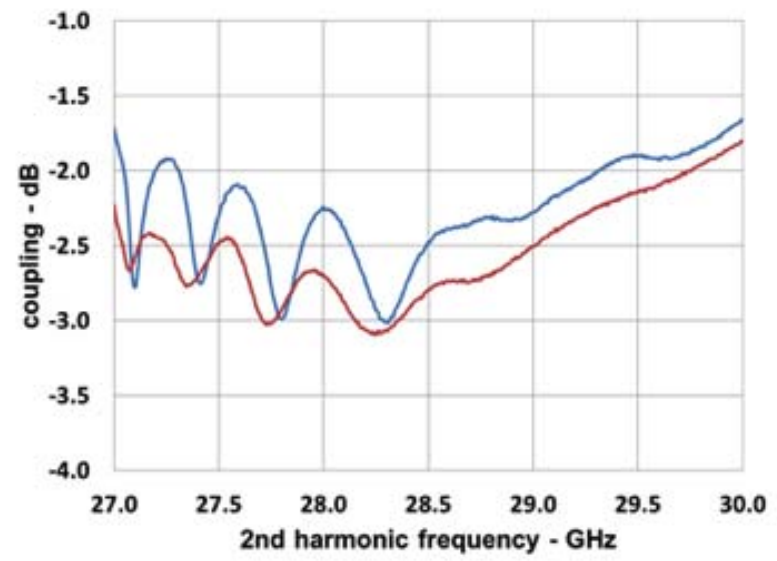

Fig. 3 Measured coupling of the two MDCs.

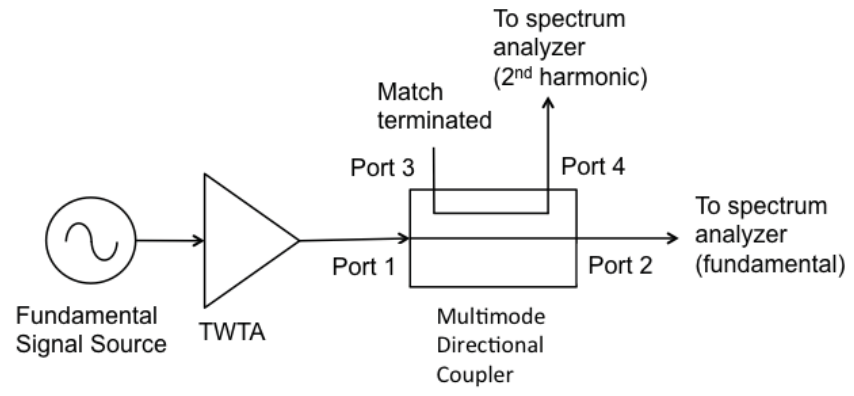

Fig. 4 Test setup for measurement of power at the fundamental and the second harmonic frequencies at the MDC output ports.

Fig. 3 compares the measured coupling for two MDC. The difference in coupling is $0.25 \mathrm{~dB}$ between the two MDCs. Fig. 4 shows the test setup used for the measurement of power at the fundamental and the second harmonic frequencies at the output ports of the MDC, when the TWTA is operating at saturation and maximum efficiency. The measured power at the fundamental frequency is uniform throughout the 13.5 to 15.0 $\mathrm{GHz}$ band and is shown in Fig. 5. The corresponding measured power at the second harmonic frequencies is shown in Fig. 6. The test data indicates that there is a significant amount of power in the second harmonic. Similar results were obtained for both MDCs. It is worth mentioning that the fundamental signal is below the cutoff frequency of the Ka-band secondary waveguide and hence propagates unperturbed in the $\mathrm{Ku}$-band primary waveguide, which is a major advantage over traditional harmonic filters.

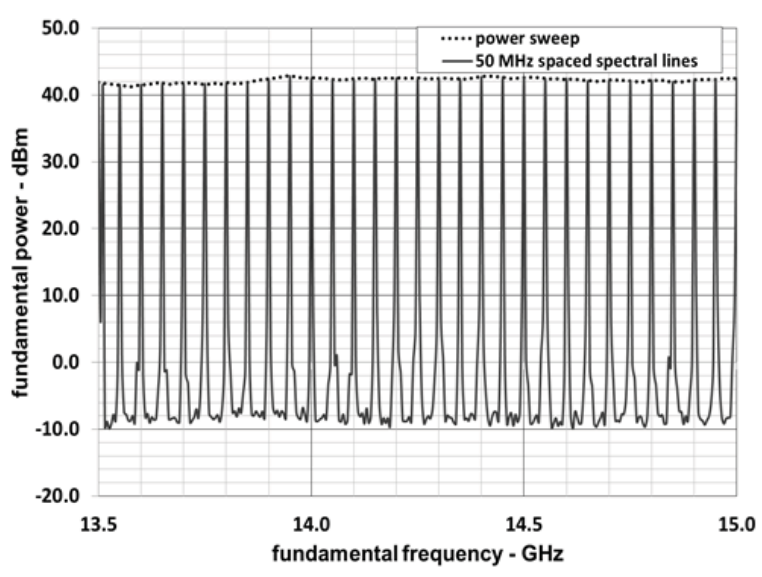

Fig. 5 Measured power at the fundamental frequencies at port 2 of the multimode directional coupler.

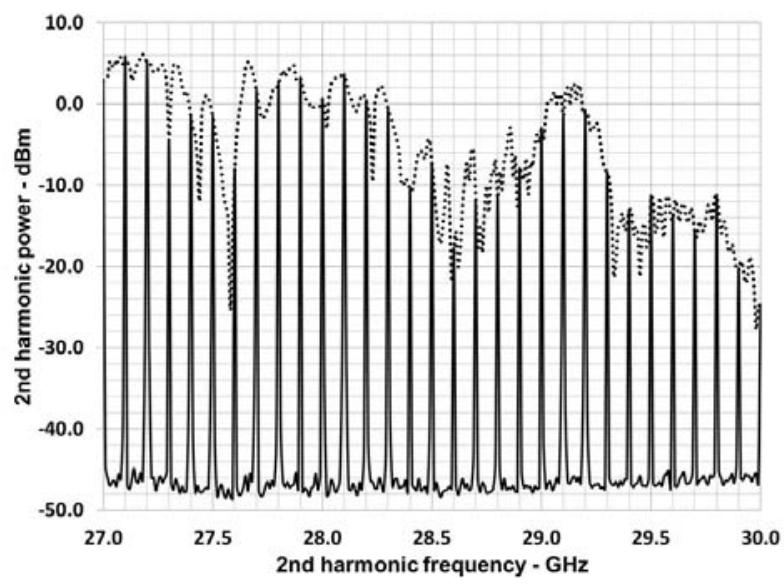

Fig. 6 Measured power at the second harmonic frequencies at port 4 of the multimode directional coupler.

\section{CONCLUSIONS}

The design, fabrication and characterization of a novel waveguide MDC is presented. The MDC design can be scaled to higher fundamental/secondary frequencies. In addition to accurate power measurements at harmonic frequencies, a potential application of the MDC is in a space borne beacon source for atmospheric propagation studies at millimeter-wave frequencies.

\section{ACKNOWLEDGEMENT}

The support from the 2012 NASA GRC Center Innovation Fund (CIF) is gratefully acknowledged.

\section{REFERENCES}

[1] Patent Application Filed with the U.S.P.T.O.

[2] R.N. Simons, J.D. Wilson, and D.A. Force, "High power and efficiency space traveling-wave tube amplifiers with reduced size and mass for NASA missions," 2008 IEEE MTT-S International Microwave Symposium Digest, pp. 319-322, Atlanta, GA, June 15-20, 2008.

[3] R.N. Simons and E.G. Wintucky, "Q-Band (37-41 GHz) Satellite Beacon Architecture for RF Propagation Experiments," 2012 IEEE Antennas and Propagation International Symposium Digest, Chicago, IL, July 8-14, 2012. 
